\title{
DEL HACER DE LAS OLLAS. LA \\ PRODUCCIÓN CERÁMICA EN UNA BASE \\ RESIDENCIAL DE LA ALDEA PIEDRA NEGRA \\ (I MILENIO DC). LAGUNA BLANCA
}

\author{
Valeria Elizabeth Espiro ${ }^{1}$
}

\section{* Introducción}

Resumen

En este trabajo se presentan las secuencias de procesos de manufactura identificadas en los materiales cerámicos provenientes de la base residencial PIN o2 de la Aldea Agroalfarera Piedra Negra, ubicada en el distrito Laguna Blanca, Belén, Catamarca. En la realización de nuestro análisis, partimos de entender los materiales cerámicos como un producto socialmente construido y a la secuencia de procesos como la sucesión de técnicas necesarias para producir un objeto cerámico. De este modo, apoyándonos en observaciones macroscópicas, submacroscópicas y microscópicas, pudimos reconstruir las secuencias de procesos técnicos empleados en parte de los materiales procedentes

de la Aldea Piedra Negra. La identificación y descripción de estas secuencias de procesos se constituye en un paso previo indispensable para la determinación de una tradición alfarera local en Laguna Blanca.

Palabras claves: cerámica - tecnología - manufactura - secuencia de procesos.

In this article we present the sequence of manufacturing processes identified in the ceramics from household PIN 02 of the Aldea Agroalfarera Piedra Negra, Laguna Blanca Belén, Catamarca. In our analysis, the ceramics is considered as a material socially constructed and as a sequence of processes such as the succession of techniques needed to produce a ceramic object. Relying on macroscopic, submicroscopic and microscopic observations, we were able to reconstruct the sequence of technical processes used in part of materials from the Aldea Piedra Negra. The identification and description of these sequences of processes is an indispensable prerequisite for establishing a local pottery tradition in Laguna Blanca.

Key words: ceramics - technology - manufacture - sequence of processes.

Recibido: agosto 2009. Aceptado: diciembre 2011.
En los últimos años, los materiales cerámicos recuperados mediante trabajos arqueológicos en la región puneña de Laguna Blanca (Dpto. Belén, Catamarca, Argentina; Figura 1) han sido el centro de nuestro interés arqueológico (Espiro 2006, 2007, 2008).

Dentro de nuestra investigación, nos ha llamado particularmente la atención, la importancia que se le otorgó a los materiales cerámicos al momento de realizar interpretaciones sobre el pasado de Laguna Blanca, no obstante los mismos nunca habían sido sometidos a análisis tecnológicos específicos. Aquí debemos mencionar que dos sucesos fueron de importancia para que estos materiales hayan sido tomados en cuenta por numerosos arqueólogos y arqueólogas al momento de hablar del pasado de esta región. El primero de estos acontecimientos se relaciona con la visita realizada durante los años 1923 y 1924 a Laguna Blanca por la expedición Muñiz Barreto a cargo del Ing. Vladimiro Weisser. Los resultados de esta expedición se cuentan en 102 enterratorios excavados, de los cuales extrajo 101 objetos cerámicos -vasijas cerámicas en su mayoría (Weisser 1923-24). Estos objetos y las libretas de campo de la expedición se encuentran en el Museo de Ciencias Naturales de la Universidad Nacional de la Plata.

Posteriormente, ocurrió en 1955 la visita de Alberto Rex González, como integrante de una comisión realizada por la Sociedad Geográfica de Americanistas. No obstante, a pesar de que González realizara prospecciones y excavaciones durante su viaje a Laguna Blanca, nunca

${ }^{1}$ CONICET, EDA e InIP - UNCa, ARGENTINA. Email: valespiro@yahoo.com.ar. 


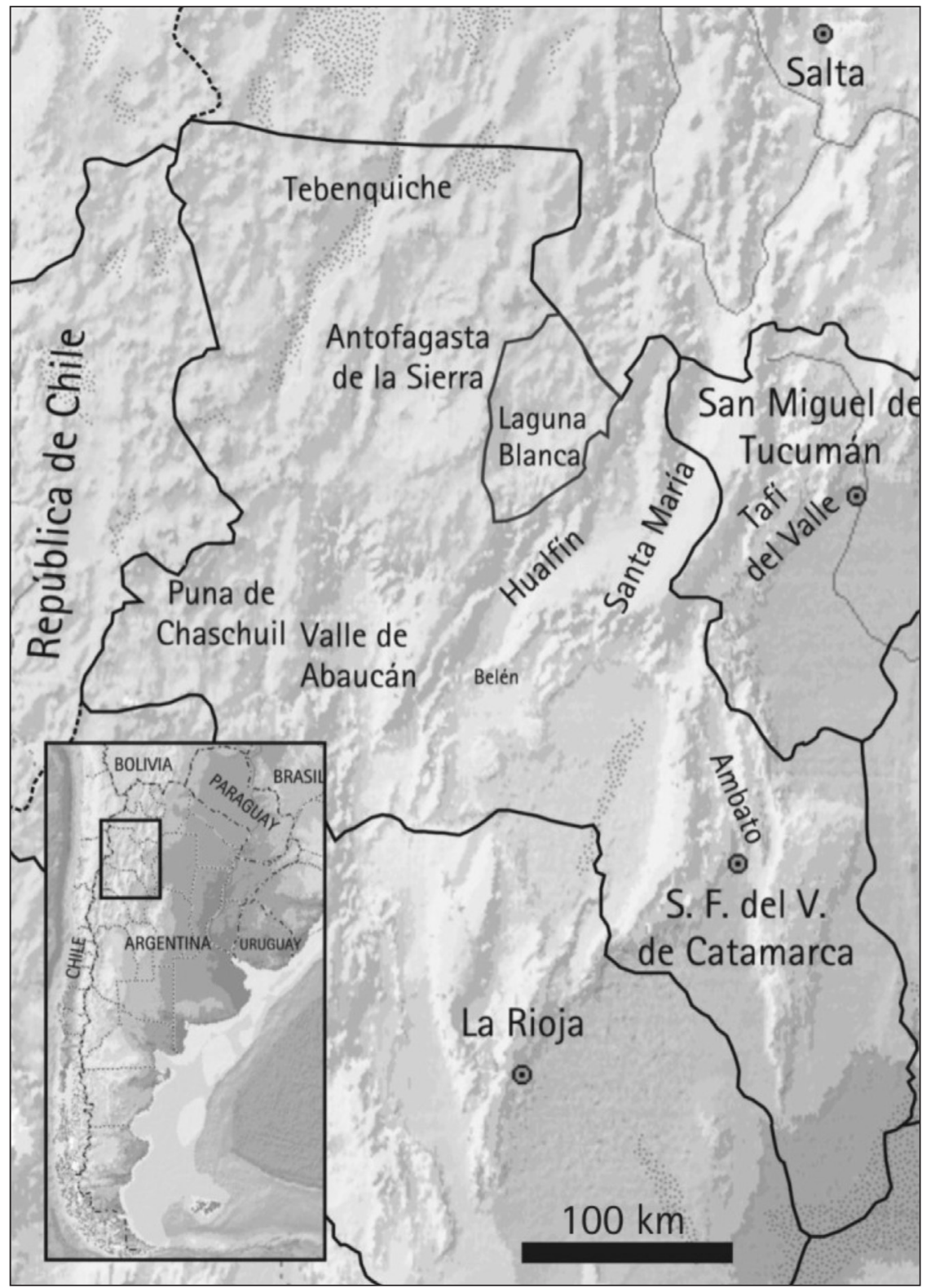

Figura 1. Ubicación del Bolsón de Laguna Blanca.

publicó los resultados de sus trabajos y lo único que conocemos sobre ellos proviene de menciones posteriores en artículos de este autor (González 1955, 1963).

De esta manera, tanto los objetos cerámicos provenientes de Laguna Blanca depositados en el Museo de La Plata, como las menciones realizadas por González, comenzaron a ser revisados por los arqueólogos y arqueólogas interesados en el pasado del área puneña. Ese material llamó la atención especialmente de aquellos interesados en explicar el modo en el cual se manifestó la producción, la circulación y el consumo de bienes en el pasado de las sociedades del Área Andina Meridional. Así, Laguna Blanca fue integrada de manera solvente a redes sociales o circuitos de intercambio dentro de diferentes modelos teóricos para interpretar estas relaciones. Podemos mencionar 
aquí el modelo de Movilidad Giratoria para patrones de tráfico e interacción económica de Lautaro Núñez y Tom Dillehay (1995 [1978]) y el relativo a la Historia de los Pueblos Circumpuneños de Myriam Tarragó (1984) (Delfino 1997, 1999). En ambos modelos, se interpreta que la presencia de alfarerías identificadas bajo los estilos Ciénaga, Candelaria, Condorhuasi y Aguada (atribuidos a los grupos de los valles mesotermales), y alfarerías San Pedro Negro Pulido (atribuidos a los grupos atacameños) serían las evidencias de tales intercambios e interrelaciones, siendo la cerámica misma uno de los objetos intercambiables.

En relación a esta construcción, los investigadores e investigadoras que han trabajado con, o mencionaron a, los materiales cerámicos provenientes de Laguna Blanca, mantuvieron como supuesto implícito la ausencia de una producción local y, en consecuencia, de una tradición alfarera en la región (González 1961-1964, 1979; Albeck y Scatollín 1984; Tarragó 1984; Scatollín y Bugliani 2005). A partir de estos supuestos, la presencia de materiales cerámicos en Laguna Blanca atribuibles a estilos decorativos del primer milenio se explicó siempre como el resultado de la participación de los pobladores de esta región en tales redes de intercambio, por lo que las cerámicas resultaban siempre de procedencias foráneas (González 1963, 1979; Scatollín y Bugliani 2005).

Sin embargo, en nuestros estudios sobre cerámicas arqueológicas de Laguna Blanca, hemos detectado una correlación entre la geología y litología local y las materias primas empleadas en la manufactura de la mayor parte de los conjuntos cerámicos, así como la presencia en los sitios de residencia de instrumentos y elementos asociados a la manufactura cerámica (Espiro 2007, 2008). Estas evidencias, sumadas a otros datos, nos sugieren que, así como en la actualidad, en el Bolsón puneño de Laguna Blanca durante mediados del primer milenio DC existía una manufactura local de materiales cerámicos.

Por esta razón, nos resultó de suma importancia comenzar con el análisis y descripción de las secuencias de producción (Rye 1988) de los materiales cerámicos. La identificación y descripción de estas secuencias de procesos se constituye en un paso previo indispensable para determinar la existencia o no de una tradición alfarera local en Laguna Blanca y este artículo representa una primera aproximación sobre el tema.

\section{* Aspectos Teóricos y Metodológicos}

En nuestro trabajo, hemos considerado a los materiales cerámicos como constituyentes de la cultura material de una sociedad. Entendemos a la cultura material como un producto socialmente construido, no solo desde las técnicas y procedimientos involucrados en la manufactura, sino contemplando los usos y concepciones que la sociedad realiza sobre los mismos (Shanks y Tilley 1987; Bate 1998).

A su vez, entendemos que el proceso de producción implica conocimientos tecnológicos. Es decir, conocimientos específicos sobre los gestos y acciones necesarios para llevar adelante la transformación de los materiales involucrados, mediante el uso de determinados instrumentos, con el fin de lograr el bien o producto buscado (Lemonnier 1992).

Lemonnier (1992) plantea que cada técnica está compuesta por cinco elementos: 1) materia; 2) energía; 3) objetos o instrumentos; 4) gestos o acciones que se agrupan en secuencias operacionales o técnicas; y 5) conocimientos específicos que incluyen los "saber como", las categorías y las representaciones. El autor afirma que las tecnologías son fenómenos sociales y una parte de esta dimensión social se observa en las elecciones tecnológicas que realiza cada sociedad. Muchas de estas elecciones tecnológicas pueden ser identificadas observando las variaciones en los cinco elementos de las tecnologías, pero las más interesantes son las que él llama "operaciones estratégicas", vale decir, aquellas que: 1) no pueden ser retrasadas; 2) no pueden ser reemplazadas; y 3 ) no pueden ser canceladas. Son las elecciones tecnológicas realizadas en estas operaciones, las que van a identificar la tecnología particular de una sociedad (Lemonnier 1992), que nosotros podemos denominar tradición tecnológica.

Ahora bien, la secuencia de técnicas necesarias para la producción de materiales cerámicos implican, entre otros procesos, atributos y técnicas: 1) obtención de las materias primas; 2) preparado de la pasta; 3 ) levantado de la pieza; 4) decoración en pasta fresca; 5) secado; 6) decoración en pasta seca; 7) tratamiento de la superficie; 8) cocción; y 9) decoración post-cocción (Shepard 1965; Cremonte 1983-1985, Rye 1988; Sinopoli 1991; Orton et al. 1993). Estas técnicas y atributos son las que hemos 


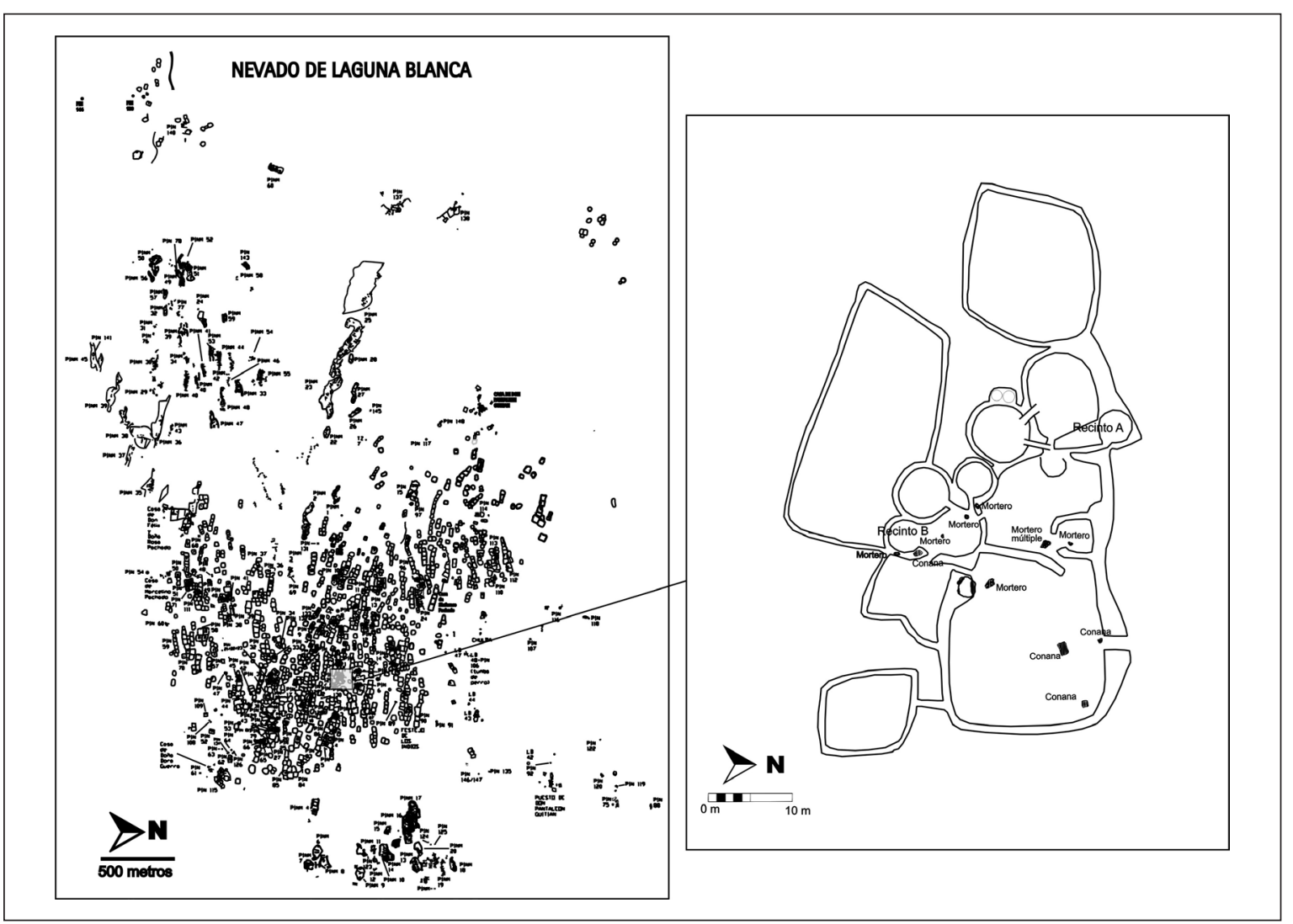

Figura 2. Plano de la Aldea Piedra Negra y detalle de PIN o2.

tenido presentes a la hora de reconstruir la secuencia de producción de los materiales analizados.

En cuanto al proceso de producción cerámica y la identificación de una manufactura local, se tuvieron en cuenta los diferentes acercamientos que diversos autores proponen (Rice 1987; Orton et al. 1993). Para la realización de nuestro estudio, se trabajó sobre la base de la distribución y presencia de materias primas cerámicas, los estilos cerámicos presentes, la identificación de artefactos relacionados a la manufactura y la aplicación de análisis tecnológicos y petrográficos sobre los materiales cerámicos.

\section{* Materiales y Métodos}

En la identificación de las secuencias de producción y/o procesos de producción (Rye 1988), se tomó como punto de partida la caracterización tecnológica y la descrip- ción de los procesos de manufactura identificados en los materiales cerámicos procedentes de la excavación

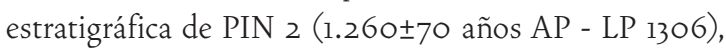
perteneciente a la Aldea Agroalfarera Piedra Negra (Figura 2) (Delfino 1997, 2005; Delfino et al. 2007).

En la actualidad, esta aldea es atravesada por tres cursos de agua de régimen permanente, varios estacionales y escorrentías temporarias. Geomorfológicamente, se ubica en el piedemonte de la Sierra de Laguna Blanca, el cual está constituido por materiales aluvionales y fluviales, en su mayoría granitos migmatíticos de colores grises y ectinitas o gneiss provenientes de la Sierra de Laguna Blanca, perteneciente a la Formación Chango Real, ${ }^{2}$ y algunas tobas

\footnotetext{
2 La Formación Chango Real se corresponde con la mayoría de las sierras que marcan los límites de nuestra área de estudio, entre ellas, la Sierra de Laguna Blanca. Según Turner (1973: 27), la formación está constituida por granitos migmatíticos, vale decir, sedimentos metamorfizados con penetración ígnea. En general,
} 


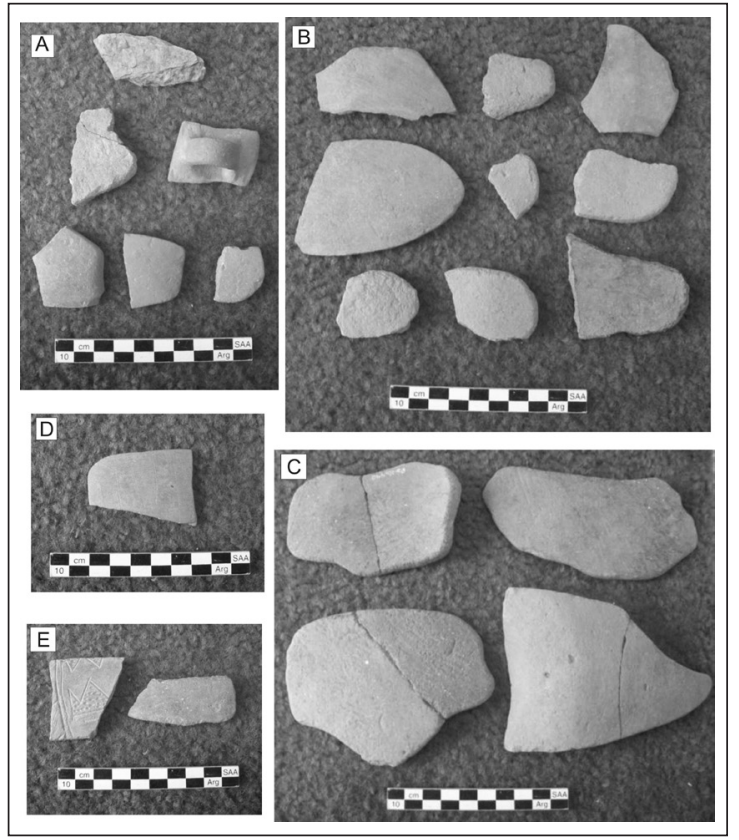

Figura 3. Algunos de los conjuntos de alisadores recuperados de PIN 15 (A), PIN o2 (B y C), PIN 36 (D), y PIN ol (E).

dacíticas e ignimbritas provenientes de los morros Ojo de Agua y El Chorro, Formación Laguna Blanca. ${ }^{3}$ También hay presencia de clastos (pegmatitas, aplitas y cuarzos) pertenecientes a los diques de rocas hipabísales que se encuentran en la Sierra de Laguna Blanca (Espiro 2008).

Podemos mencionar, para esta aldea, el hallazgo de instrumentos asociados a la manufactura cerámica (alisadores y pulidores cerámicos y líticos, restos de pigmentos, restos de pizarras, arcillas cocidas, etc.) en muchas de las

estas rocas son homogéneas, de color rojizo o grisáceo. Macroscópicamente, la roca es de grano grueso a porfiroideo, mostrando microclino, plagioclasas, cuarzo, biotita, moscovita y como minerales accesorios o secundarios circón, apatita, clorita y epidoto. La pasta por lo general es de cuarzo o feldespato.

3 La Formación Laguna Blanca está constituida principalmente por tobas dacíticas e ignimbritas. Estas rocas se apoyan discordantemente tanto sobre los granitos migmatíticos de la Formación Chango Real como sobre las ectinitas de la Formación Loma Corral. Los elementos que integran esta Formación son de color predominante gris en corte fresco y rojizo claro por meteorización. Las tobas vitrocristalinas presentan clastos de dimensiones variables, los cuales son en su mayoría dacitas con presencia de granitos migmatíticos y de ectinitas (Turner 1973: 36).

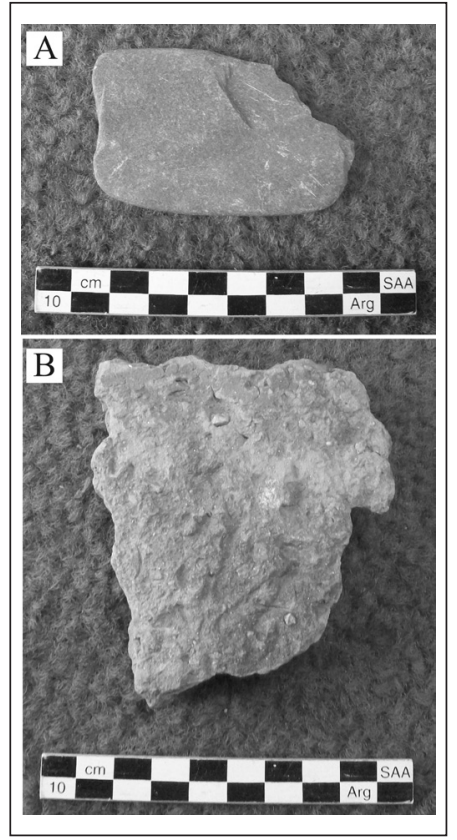

Figura 4. Pizarra con adherencias de arcillas (A) y fragmento de arcilla consolidada (B).

bases residenciales con ocupaciones contemporáneas a PIN O2 (Delfino et al. 2012), como en PIN ol (1270 \pm 80 años AP - LP 2622), PIN 10 (1100 \pm 60 C14 AP - LP 2473), PIN 15 (1170 \pm 80 C14 AP - LP 2450), PIN 23 (1250 \pm 80 $\mathrm{C}_{14} \mathrm{AP}$ - LP 2451), PIN 36 (1480 \pm 50 C14 AP - LP 2572) y PIN 40 (1210 $\pm 80 \mathrm{C}_{14}$ AP - LP 2474), evidencias que nos sugieren que sus miembros participaron, al menos en algunos momentos, de la secuencia de producción alfarera (Figuras 3 y 4 ).

Piedra Negra o2 (PIN O2) se ubica en el centro de la Aldea Piedra Negra y es una base residencial (Manzanilla 1986) integrada por nueve recintos subcirculares adosados y distribuidos alrededor de tres recintos mayores de planta cuadrangular, y por tres recintos mayores de planta cuadrangular. En esta unidad habitacional, se han excavado estratigráficamente por área abierta dos recintos (A y B). Los recintos excavados en PIN o2 fueron construidos con paredes de roca disponible en el mismo piedemonte, según hiladas simples y con aparejos rústicos. Se precisó que la resolución de los recintos implicó la remoción de tierra para lograr el nivel negativo de su interior, particularidad constructiva que recuerda a las 


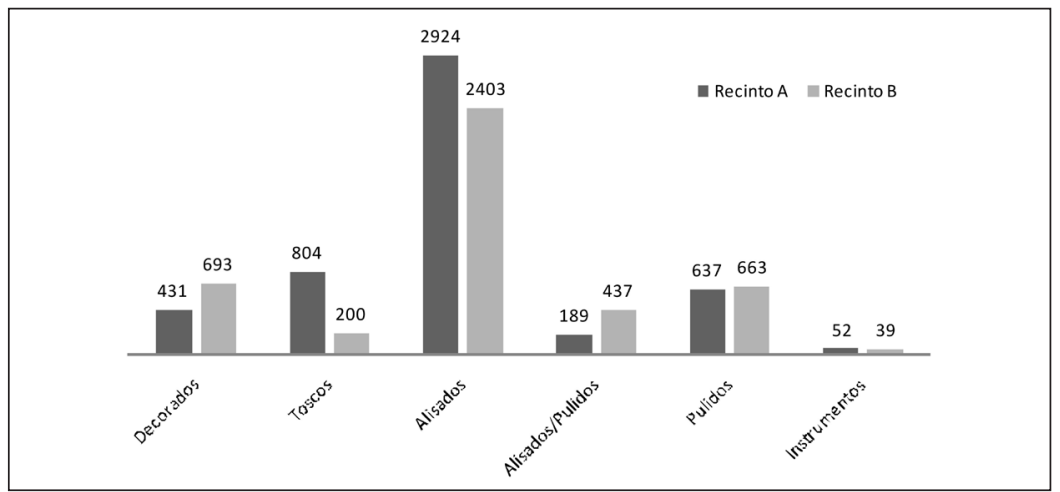

Figura 5. Frecuencia de fragmentos e instrumentos recuperados en los recintos A y B de PIN o2.

descripciones de casas pozo y/o semi-pozo definidas por González para el valle de Hualfín (Delfino 1997, 2005).

Entre las evidencias artefactuales, recuperadas en el recinto $\mathrm{A}$, se destacan varios objetos de metal (en cobre y oro), además de artefactos en hueso, cientos de restos óseos faunísticos, gran cantidad de material lítico (abundantes lascas e instrumentos confeccionados en diversas materias primas) y más de cinco mil fragmentos cerámicos (Delfino 1999; Delfino et al. 2007). Las características presentes en los materiales recuperados, la presencia de una estructura de combustión, de un muro deflector construido en la entrada y de varios instrumentos de molienda en el interior del recinto (manos de moler, conanas y morteros), nos llevaron a interpretar a este espacio intramuros como destinado a la realización de actividades domésticas. Se consideró que en el mismo se llevaba a cabo la preparación, cocción y consumo de alimentos, así como otras actividades productivas y reproductivas. Entre las diversas actividades, se presume también el consumo de sustancias psicoactivas, debido al alto número de hallazgos de fragmentos de pipas cerámicas. En cuanto al Recinto B, se puede decir que presenta diferencias sustanciales con el anterior. En primer lugar, los restos óseos son escasos, no se hallaron instrumentos de molienda en su interior y no posee estructura de combustión, ni deflector. El material cerámico es abundante, pero menor al recuperado en el recinto A, y la proporción de materiales con presencia de decorado en superficie y pulido son superiores en el recinto B. En lo referente a los instrumentos hallados, observamos una mayor recurrencia de fichas y "muyunas" en el Recinto $\mathrm{A}$, y una mayor proporción de alisadores cerámicos en el
Recinto B (Figura 5). Estas diferencias nos podrían estar indicando la realización de prácticas domésticas distintas en cada uno. Hemos considerado una sincronicidad en la ocupación de ambos recintos, a pesar de la ausencia de fechados absolutos para el recinto $\mathrm{B}$, ya que durante el trabajo de reconstrucción física de los materiales cerámicos obtuvimos ensambles de fragmentos que proveían de la estratigrafía de ambos recintos (Espiro 2008).

Para la caracterización de las secuencias de procesos involucrados en los materiales cerámicos, objeto de nuestro estudio, realizamos una revisión de la muestra de 1.019 fragmentos cerámicos pertenecientes al recinto A del PIN 2, objeto de nuestro estudio tecnológico previo (Espiro 2006, 2008). Este estudio fue realizado por medio de la observación en lupa binocular estereoscópica y en microscopio petrográfico de cortes delgados cerámicos. Nos parece importante recordar que la muestra se seleccionó mediante un muestreo estratificado aleatorio (Shennan 1992) a partir de los 5.037 fragmentos recuperados del recinto A, cuya estratificación se correspondió a los tres grandes grupos observados: 1) decorados (por agregado de pintura, por corte de la superficie externa, por agregado y/o modelado de arcilla), 2) no decorados y 3) instrumentos (torteros y/o "muyunas", tembetaes, orejeras, fichas, alisadores, etc.).

En busca de nuestro objetivo, tomamos como punto de partida los seis tipos de pastas identificados previamente para el PIN o2; Pastas A, B1, B2, C, D y E (Espiro 2006); cuyas características generales se resumen en las Tablas 1,2 y 3 . 
Del hacer de las ollas. La producción cerámica en una base residencial de la Aldea Piedra Negra...

\begin{tabular}{|c|c|c|c|c|c|c|}
\hline \multirow{2}{*}{ Pasta } & \multicolumn{2}{|c|}{ Matriz } & \multicolumn{4}{c|}{ Porosidad de la Matriz } \\
\cline { 2 - 7 } & Textura & Color & Porcentaje & Forma & Tamaño & Orientación \\
\hline A & Mixta & Uniforme & Baja & irregular & Uniforme & Homogénea \\
\hline B1 & Mixta & No uniforme & Baja, media & irregular & No uniforme & Heterogénea \\
\hline B2 & Microgranosa & Uniforme & Baja, media & irregular & No uniforme & Homogénea \\
\hline C & Lepidoblástica & No uniforme & Baja, media & irregular & No uniforme & Heterogénea \\
\hline D & Microgranosa & Uniforme & Baja & irregular & uniforme & Heterogénea \\
\hline E & Lepidoblástica & uniforme & Media & irregular & No uniforme & Homogénea \\
\hline
\end{tabular}

Tabla 1. Características generales de la Matriz de cada tipo de Pasta Cerámica.

\begin{tabular}{|c|c|c|c|c|c|c|c|}
\hline \multirow{2}{*}{ Pasta } & \multicolumn{7}{|c|}{ Inclusiones } \\
\hline & Densidad & Tipo & Orientación & Forma & Tamaño & Ordenamiento & Litología \\
\hline A & $<20 \%$ & $\begin{array}{c}\text { Cristoclastos, } \\
\text { litoclastos }\end{array}$ & Homogénea & $\begin{array}{l}\text { Subredondeadas, } \\
\text { redondeadas }\end{array}$ & $\begin{array}{l}\text { Particular, grano } \\
\text { fino, grano medio }\end{array}$ & $\begin{array}{l}\text { Justo, bueno, } \\
\text { muy bueno }\end{array}$ & Solo volcánica \\
\hline B1 & $>30 \%$ & $\begin{array}{c}\text { Cristoclastos, } \\
\text { litoclastos }\end{array}$ & Heterogénea & $\begin{array}{c}\text { Angulares, subangulares, } \\
\text { subredondeadas }\end{array}$ & $\begin{array}{l}\text { grano fino, grano } \\
\text { medio, gránulos }\end{array}$ & Justo & $\begin{array}{l}\text { Volcánica, plutónica, } \\
\text { metamórfica }\end{array}$ \\
\hline $\mathrm{B}_{2}$ & $<30 \%$ & $\begin{array}{c}\text { Cristoclastos, } \\
\text { litoclastos }\end{array}$ & Homogénea & $\begin{array}{c}\text { Angulares, subangulares, } \\
\text { subredondeadas }\end{array}$ & $\begin{array}{l}\text { Particular, grano } \\
\text { fino, grano medio }\end{array}$ & Justo & $\begin{array}{l}\text { Volcánica, plutónica, } \\
\text { metamórfica }\end{array}$ \\
\hline $\mathrm{C}$ & $\begin{array}{l}>10 \% \\
<30 \%\end{array}$ & $\begin{array}{c}\text { Cristoclastos, } \\
\text { litoclastos }\end{array}$ & $\begin{array}{l}\text { Preferencial, } \\
\text { heterogénea }\end{array}$ & $\begin{array}{c}\text { Angulares, subangulares, } \\
\text { subredondeadas, } \\
\text { redondeadas }\end{array}$ & $\begin{array}{l}\text { Particular, grano } \\
\text { fino, grano medio }\end{array}$ & Pobre, justo & Volcánica, plutónica \\
\hline D & $<5 \%$ & $\begin{array}{c}\text { Cristoclastos, } \\
\text { litoclastos }\end{array}$ & Heterogénea & Suangulares & $\begin{array}{l}\text { Particular, grano } \\
\text { fino, grano medio }\end{array}$ & Bueno & Plutónica \\
\hline $\mathbf{E}$ & $<10 \%$ & $\begin{array}{c}\text { Cristoclastos, } \\
\text { litoclastos }\end{array}$ & Homogénea & $\begin{array}{l}\text { Subangulares, } \\
\text { subredondeadas }\end{array}$ & $\begin{array}{l}\text { Particular, grano } \\
\text { fino, grano medio }\end{array}$ & justo & $\begin{array}{l}\text { Metamórfica, } \\
\text { sedimentária }\end{array}$ \\
\hline
\end{tabular}

Tabla 2. Características generales de las inclusiones de cada tipo de Pasta Cerámica.

\begin{tabular}{|c|c|c|c|c|}
\hline \multirow{2}{*}{ Pasta } & \multicolumn{2}{|c|}{ Cristoclastos } & \multicolumn{2}{|c|}{ Litoclastos } \\
\hline & Predominantes & Aislados & Predominantes & Aislados \\
\hline A & $\begin{array}{l}\text { Cuarzos, angioclasas, biotitas, muscovitas, } \\
\text { feldespatos alterados, hornblendas }\end{array}$ & $\begin{array}{l}\text { Inclusiones pardo rojizas, ftanita, microclino, } \\
\text { feldespato calcosódico, anfíboles }\end{array}$ & $\begin{array}{l}\text { Vidrios volcánicos, esferulitas, } \\
\text { vulcánitas, andesitas }\end{array}$ & $\begin{array}{l}\text { Gránulos de arcilla, } \\
\text { inclusión anisótropa }\end{array}$ \\
\hline B & $\begin{array}{c}\text { Cuarzos, angioclasas, biotitas, ortoclasas, } \\
\text { hornblendas, minerales opacos, } \\
\text { muscovitas, feldespatos alterados, } \\
\text { microclinos }\end{array}$ & $\begin{array}{l}\text { Piroxenos, anfíboles, turmalina, granates, } \\
\text { épidoto, circón, microclinos, pertíticos, } \\
\text { plagioclasas zoneadas, cuarzos con extinción } \\
\text { ondulosa, minerales muy sericitizados }\end{array}$ & $\begin{array}{l}\text { Metacuarcita, rocas } \\
\text { plutónicas, granulos de arcilla, } \\
\text { vidrios volcánicos, esferulitas }\end{array}$ & $\begin{array}{l}\text { Andesitas, illitas, } \\
\text { metamórficas }\end{array}$ \\
\hline $\mathrm{C}$ & $\begin{array}{l}\text { Cuarzos, plagioclasas (oligoclasas), } \\
\text { muscovitas, biotitas, plagioclasas } \\
\text { zoneadas, microclinos, feldespatos } \\
\text { alterados }\end{array}$ & $\begin{array}{c}\text { Hornblendas verdes, granates, apatita, } \\
\text { microclino pertíticos, minerales opacos, } \\
\text { anfíboles }\end{array}$ & $\begin{array}{l}\text { Vidrio volcánico perlitico, } \\
\text { vulcanitas no identificadas, } \\
\text { rocas plutónicas }\end{array}$ & $\begin{array}{l}\text { Andesitas, gránulos } \\
\text { de arcilla }\end{array}$ \\
\hline $\mathrm{D}$ & $\begin{array}{c}\text { Cuarzo, biotitas, plagioclasas, muscovitas, } \\
\text { feldespatos alterados }\end{array}$ & Afíbol, microclino & Rocas plutónicas & \\
\hline E & $\begin{array}{c}\text { Cuarzos, plagioclasas (con maclas según } \\
\text { ley de albita y maclas de dos individuos), } \\
\text { biotitas, muscovitas }\end{array}$ & $\begin{array}{l}\text { Hornblendas verdes, microclinos, minerales } \\
\text { sericitizados, cuarzos con extinción zoneada, } \\
\text { mineral de hierro, feldespato potásico pertitico }\end{array}$ & Limonitas & $\begin{array}{l}\text { Metacuarcitas, } \\
\text { gránulos de arcilla }\end{array}$ \\
\hline
\end{tabular}

Tabla 3. Identificación de las inclusiones cada tipo de Pasta Cerámica. 
La pasta A presenta una compactación alta a muy alta con baja frecuencia de cavidades; la cocción se presenta regular y en la mayoría de los casos reductora, macroscópicamente se observan muy pocas inclusiones blancas y laminillas de muscovitas. Al microscopio petrográfico, las inclusiones no sobrepasan tamaños mayores a 0,25 mm y no superan el $20 \%$ en abundancia, los minerales más representados son cristales de cuarzos, plagioclasas, micas y solo se observaron litoclastos de origen volcánicos como vidrios volcánicos (de textura fluidas, esferulítica con vesículas y eutaxica; Figura 6), andesitas (con textura vitrocristalina e inclusiones de plagioclasas; ver Figura 6) y vulcanitas no identificadas (de textura vitrocristalina y criptocristalina).

La Pasta B1 posee compactación media, pudiendo ser de cocción regular e irregular, oxidante reductora o mixta. Las inclusiones de micas, cuarzos, feldespatos y litoclastos visibles macroscópicamente se presentan en una densidad igual o mayor al 30\% y de forma, tamaños y distribución heterogéneos. La Pasta B2 presentó una compactación alta y muy alta, la cocción en la mayoría de los casos es reductora pero en algunos casos se presentó mixta y la densidad de inclusiones no supera el 20\%. Al microscopio petrográfico las Pastas B1 (Figura 7) y B2 muestran inclusiones de diversos orígenes litológicos dentro de los mismos cortes. Podemos mencionar ente los minerales más abundantes los cristales de cuarzo, plagioclasas, micas y litoclastos de origen granítico, así como vidrios volcánicos, andesitas, vulcanitas $y$, menos frecuentes, algunos litoclastos metamórficos como metacuarcitas y filitas.

La Pasta C presentaba macroscópicamente la característica de poseer mucha mica, presentar una compactación media y alta y su cocción es tanto oxidante como reductora. Al microscopio petrográfico, se observaron inclusiones de origen plutónico y volcánico, de tamaños heterogéneos, llegando en algunos casos a los $3 \mathrm{~mm}$, con densidades de entre 10 y $30 \%$. Podemos mencionar entre los minerales y litoclastos más abundantes los cuarzos, plagioclasas, micas, minerales accesorios, litoclastos graníticos, vidrios volcánicos, andesitas y vulcanitas.

Podemos caracterizar a la Pasta D como de compactación baja, de una cocción irregular mixta, con densidades de inclusiones del 5\%, de tamaños medianos a gruesos. Se observó al microscopio inclusiones que mostraban una litología solo plutónica destacando minerales de cuarzos, micas, feldespatos, plagioclasas, minerales accesorios y litoclastos graníticos.

La Pasta E presentó una compactación mediana y alta, de cocción reductora en su mayoría, una densidad de inclusiones de hasta el 10\%. Presentó al microscopio minerales y litoclastos propios de ambientes metamórficos y sedimentarios, destacando los litoclastos de metacuarcita, illita, filita, lutita, limolita y abundantes gránulos de arcillas.

Nos parece importante remarcar algunos aspectos relacionados al preparado de la pasta que nos llamaron la atención. En este sentido, observamos que la Pasta A es rica en inclusiones volcánicas, como vidrios y vulcanitas, de tamaños pequeños, y en todos los casos, en formas redondeadas y subredondeadas. Las inclusiones de las Pasta B2, caracterizadas por líticos de rocas plutónicas de tamaños pequeños y de formas redondeadas, nos sugirieren su presencia como inclusiones propias de la arcilla y no debido al agregado de las mismas como materiales antiplásticos (Espiro 2008).

El gran tamaño y las formas angulosas de las inclusiones de las Pastas $\mathrm{B} 1$ y $\mathrm{C}$ nos llevan a pensar en el agregado intencional de las mismas por las/los alfareras/os. Las características de la Pasta C, de una porosidad alta e inclusiones abundantes de micas, litoclastos volcánicos y plutónicos de grano pequeño, le otorgan a la misma óptimas condiciones para la exposición al fuego (Rice 1987: 229-230; Arnold 2003: 72-80). En este sentido, la gran cantidad de fragmentos cerámicos con hollín en superficie correspondientes a esta pasta nos indican, al menos, la exposición al fuego de los materiales cerámicos de los que formaban parte. En cuanto a la cerámica confeccionada con la Pasta $\mathrm{Bl}$, con grandes y angulosos litoclastos plutónicos, que según Arnold (2003: 80) no otorgan a la pasta propiedades óptimas para su exposición al fuego, al observar los fragmentos notamos la ausencia de hollín, así como de otras marcas de exposición al fuego. De esta manera, proponemos que, en el caso de la Pasta $\mathrm{B}$, se escogieron materias primas para la confección de materiales que no estuvieron destinados a la cocción de alimentos o a la exposición prolongada al fuego (Espiro 2008). 


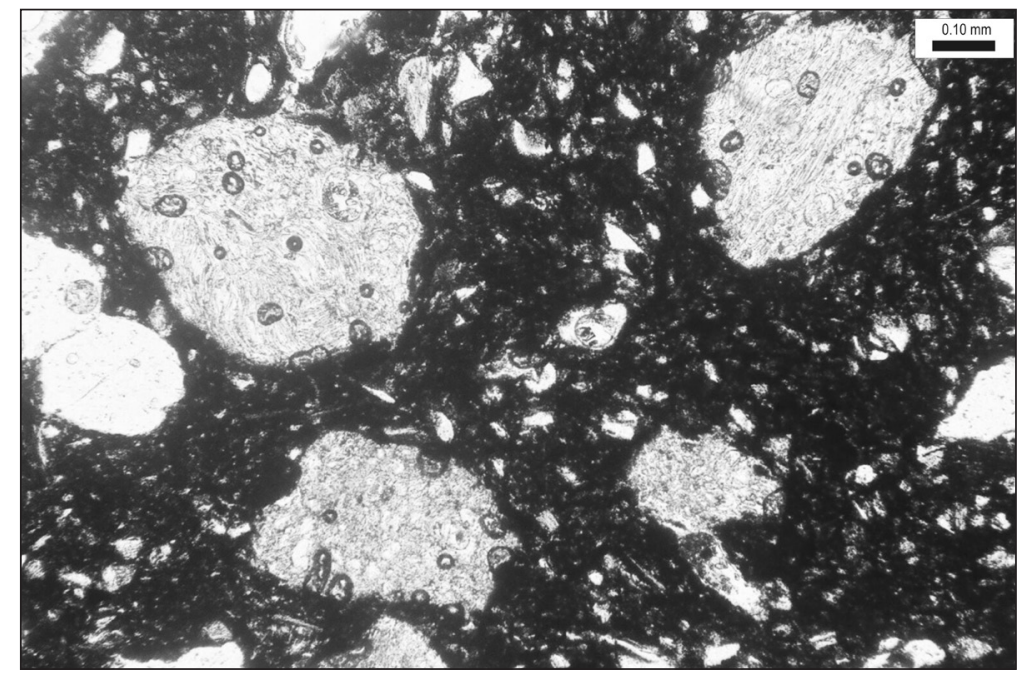

Figura 6. Fotomicrografía mostrando litoclastos redondeados de vidrios volcánicos con textura vesicular y eutáxica, característicos de la Pasta A, PPL - aumentos de 25 X.

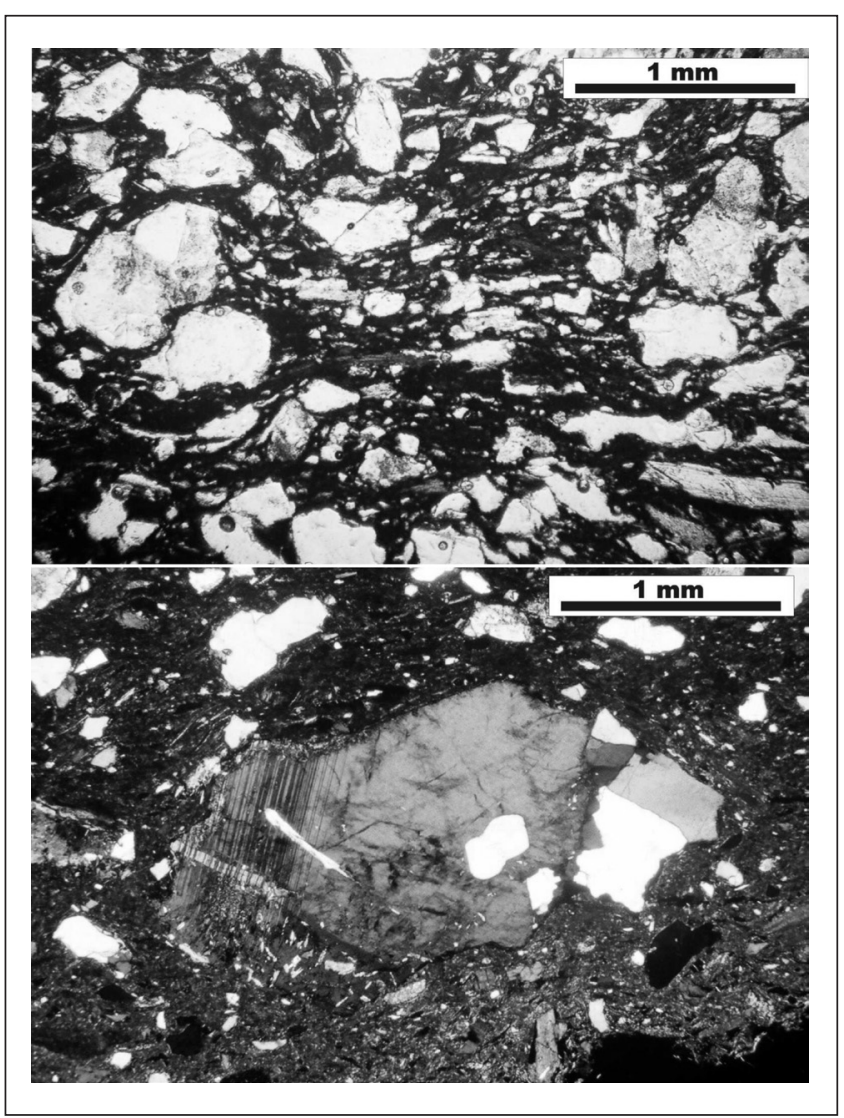

Figura 7. Fotomicrografías mostrando características de la Pasta $\mathrm{B}$ (imagen superior), PPL; e inclusión de roca granítica subangular de gran tamaño (imagen inferior), XPL; ambas figuras con aumentos de $16 \mathrm{X}$. 


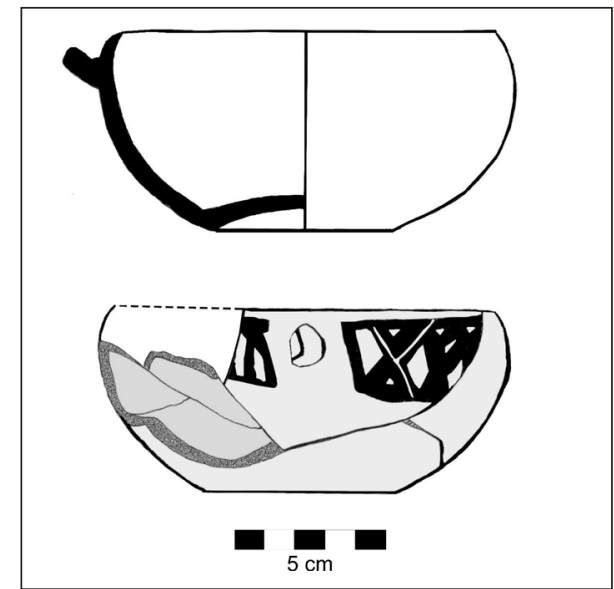

Figura 8. Reconstrucción de un puco cerámico recuperado en PIN O2.

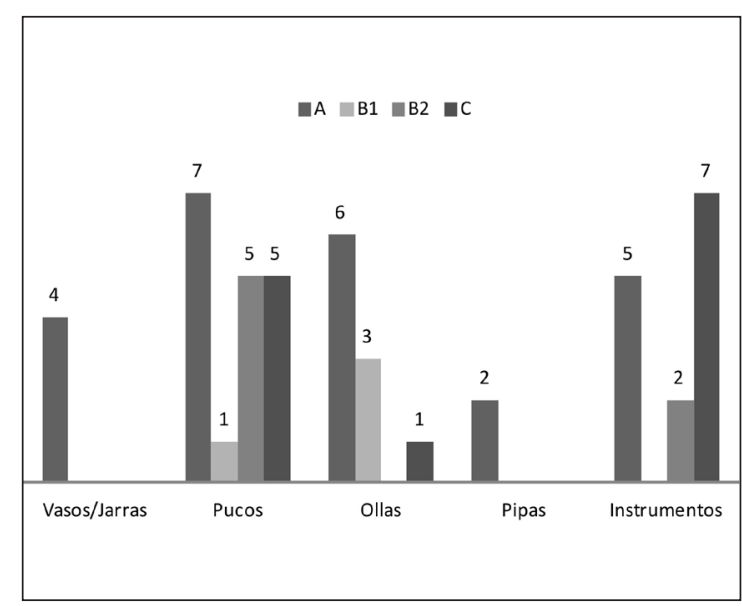

Figura 9. Cantidad mínima de objetos cerámicos identificados por tipo de pasta.

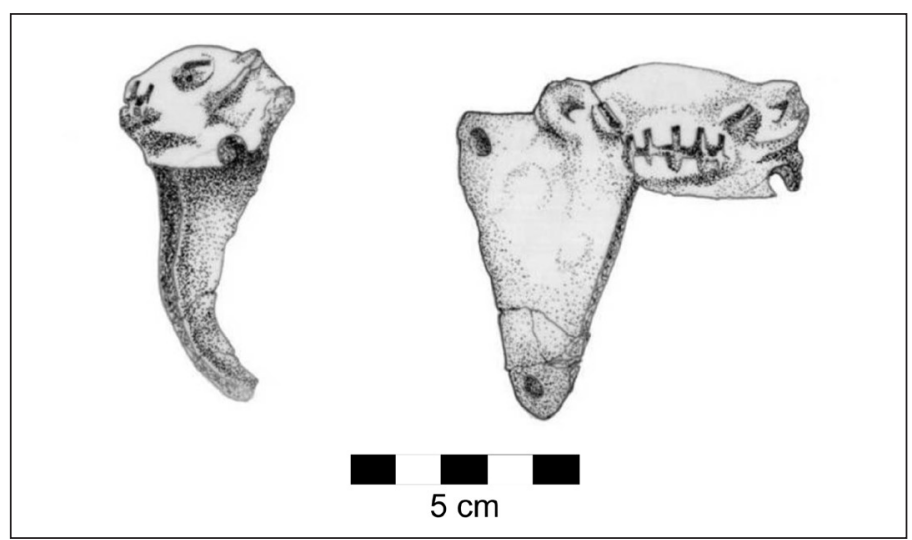

Figura 10. Fragmentos de un puco cerámico confeccionado con el tipo de Pasta A decorado mediante técnicas de incisión y modelado de figuras zoomorfas.

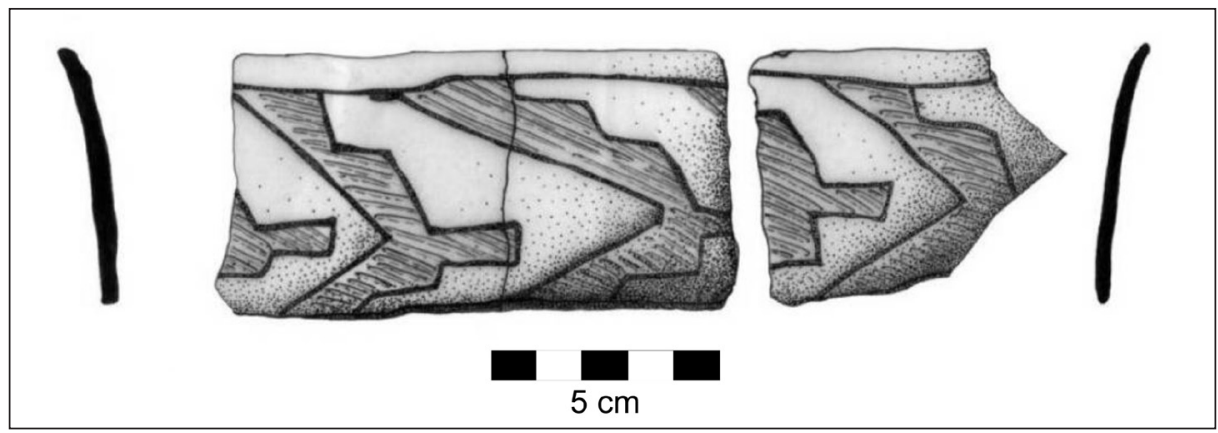

Figura 11. Fragmentos de un puco cerámico confeccionado con el tipo de Pasta A decorado mediante técnicas de incisión de motivos geométricos. 
Continuando con el objetivo del presente trabajo, para la reconstrucción de las secuencias de producción, separamos los conjuntos de fragmentos por tipos de pastas y comenzamos el trabajo de detectar e individualizar la mayor cantidad de formas de materiales cerámicos presentes en cada conjunto. La reconstrucción de formas a partir de material fragmentado constituye un gran desafío para lo cual recurrimos al agrupamiento por medio de familias de fragmentos, a la reconstrucción física parcial del material cerámico mediante el ensamblado de fragmentos (Figura 8) y, en los casos que lo posibilitaron, se procedió a la reconstrucción 3D, mediante el empleo del software Autocad 2008, con la función revolve o sólido de revoluciones (Orton et al. 1993; Contreras Córtes 1994; Zagorodny y Balesta 1999; Zagorodny 2000). En este sentido, podemos mencionar que los fragmentos de bordes son los que nos proveyeron mayor información sobre las formas, mientras que los restos de bases fueron los más indicados para precisar la cantidad de materiales cerámicos presentes. De ese modo, se pudo aislar para cada tipo de pasta la presencia de determinadas formas y la cantidad mínima de materiales cerámicos. ${ }^{4}$

Se pudieron reconstruir formas y materiales cerámicos solo en los tipos de Pasta A, B1, B2 y C. Las formas reconstruidas fueron de vaso o jarra, pucos, olla, pipa e instrumentos varios (Figura 9).

\section{$*$ Resultados}

Para los objetivos de este trabajo, hemos escogido solo las formas que se corresponden con vasijas o contenedores cerámicos para la reconstrucción de las secuencias de procesos y posterior comparación. Los instrumentos cerámicos y las pipas serán abordados en futuros trabajos.

\section{Pasta A}

Vasos y Jarras. Se pudo observar un mínimo de cuatro vasos o jarras correspondientes a la Pasta A. Para su confección, se procedió al levantado de los materiales em-

4 Debemos mencionar que de ningún modo la cantidad de objetos cerámicos detectados representa la totalidad de los que podrían haber estado en el recinto A de PIN O2, ya que, como aclaramos anteriormente, estamos trabajando con solo una muestra representativa. pleando las técnicas de modelado y rodete. Se emplearon las técnicas de pulido en tres de los casos estudiados y de bruñido en uno para el acabado de la superficie externa y para la superficie interna se emplearon las técnicas de pulido en tres y en uno de los casos no se práctico acabado de superficie. En lo que respecta a la decoración de los vasos/jarras confeccionados con la Pasta A, solo se escogió la superficie externa y en ella se practicaron técnicas decorativas por corte de la superficie. Los motivos de la decoración practicada son de líneas y líneas con círculos.

A su vez, se pudo observar la presencia de técnicas de tratamiento de superficies en algunos casos, como la presencia de baño en ambas superficies y de engobe en superficie interna con un baño en la externa. En lo que respecta al ambiente y tipo de cocción, se pudo determinar en todos los casos una cocción regular del material cerámico, así como la presencia de dos tipos de cocciones: oxidantes en dos y reductoras en los dos restantes.

Pucos o escudillas. Se detectó la presencia de siete pucos dentro de la muestra analizada de la Pasta A. Para el levantado de los mismos se empleo las técnicas de modelado y rodete. El acabado de la superficie externa se logró mediante el pulido en seis de los pucos y de bruñido en el restante, mientras que para la superficie interna se emplearon las técnicas de alisado en uno, pulido en cuatro, pulido con instrumento 5 en uno y bruñido en otro. La decoración de los pucos confeccionados con la Pasta A solo se efectúo en la superficie externa y con técnicas decorativas por corte de la superficie fresca: incisión en cuatro; incisión y estampamiento en dos; y incisión, escisión y estampamiento en uno de los casos. En algunos ejemplares, junto con la decoración por corte, se practicaron otras técnicas como el modelado y el aplique (Figura 10). Los motivos de la decoración practicada en estos pucos en dos casos son figuras zoomorfas y en los restantes se aplicó líneas y puntos; líneas, círculos y figuras zoomorfas; líneas y figuras geométricas; y solo figuras geométricas (Figura 11).

5 Consideramos que la mayoría de las técnicas de acabado de superficies debieron involucrar el empleo de al menos un instrumento o herramienta. No obstante, al realizar la aclaración de "pulido y/o alisado con instrumento" estamos haciendo alusión al hecho de que la herramienta con la cual se realizó el acabado produjo un patrón direccional de marcas, líneas o estrías en la superficie de la cerámica. Rye (1988) emplea el término de "incompletely burnished" cuando se observan estos patrones de direccionalidad en el acabado de superficie. 


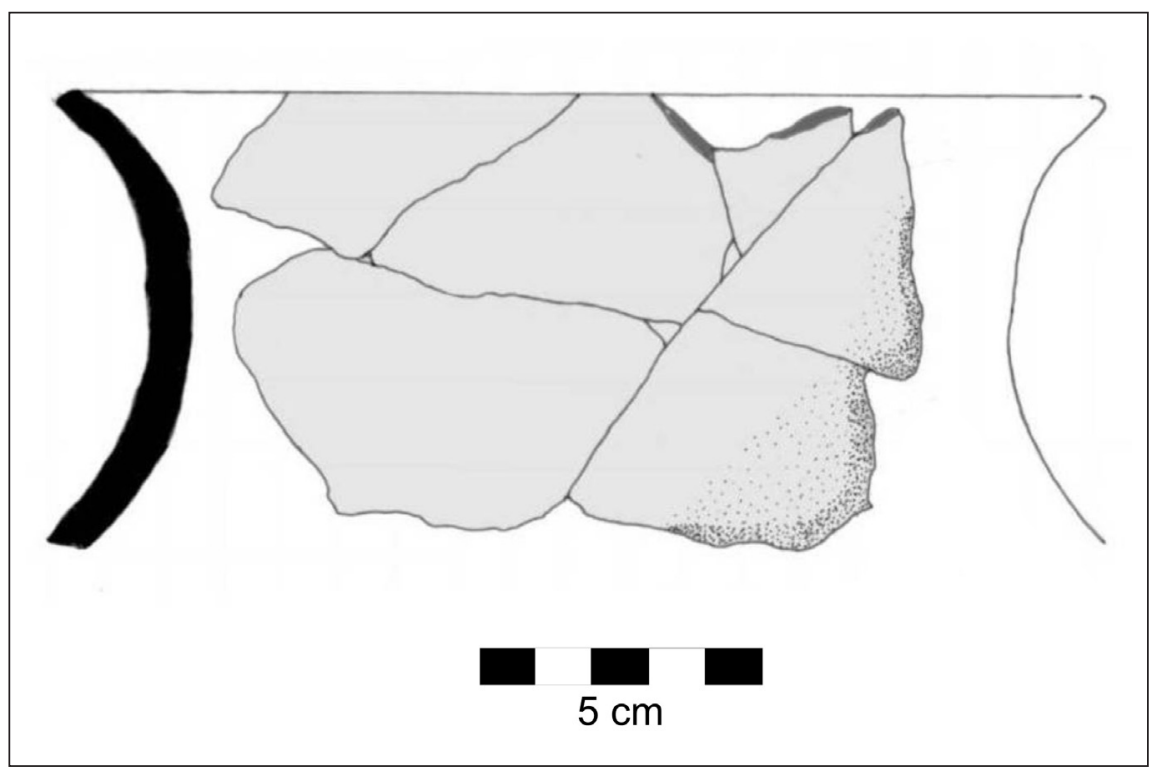

Figura 12. Fragmentos de cuello y borde de una olla confeccionada con la Pasta B1.

Se observó la presencia de técnicas de tratamiento de superficies en algunos casos; baño en ambas superficies; engobe interno en dos casos; engobe externo; y baño externo. En lo que respecta al ambiente y tipo de cocción, se pudo determinar en todos los casos una cocción regular del material cerámico, así como la presencia de dos tipos de cocciones: oxidantes en dos y reductoras en los cinco restantes.

Ollas. Se detectaron al menos seis ollas confeccionadas con la Pasta A y las mismas fueron levantadas con las técnicas de modelado y rodete. Se emplearon las técnicas de acabado de la superficie externa de alisado en cuatro ollas y pulido en dos; y para la superficie interna se emplearon las técnicas de alisado en cuatro casos, alisado con instrumento en uno y pulido en el restante. En lo que respecta a la decoración de las ollas, solo se observó en dos casos en la superficie externa y realizada con técnicas decorativas por corte de la superficie de incisión y estampamiento de líneas y círculos un caso, y peinado en otro.

A su vez, se pudo observar la presencia de técnicas de tratamiento de superficies en algunos casos, como la presencia de baño y de engobe en la superficie externa. En lo que respecta al ambiente y tipo de cocción, se pudo observar una mayor variabilidad que con los pucos; de- terminando casos con cocción regular e irregular del material cerámico, así como la presencia de diversos tipos de cocciones: oxidante en dos ollas, oxidante incompleta en una, oxidante externa-reductora interna en otra y reductora en dos.

\section{Pasta B1}

Pucos o escudillas. Dentro de la muestra analizada correspondiente a la Pasta $\mathrm{B} 1$, solo se identificó un puco, para el cual se emplearon las técnicas de modelado y rodete para su levantado, se alisaron ambas superficies del puco, no se practicó decoración ni tratamiento de superficie y fue cocido en una atmósfera reductora, presentando una cocción regular.

Ollas. Se pudo reconstruir un mínimo de tres ollas dentro del grupo de la Pasta Bi. Las técnicas de levantado de la pieza en estos casos fueron de modelado y rodete. En cuanto al acabado de la superficie externa, se observó la aplicación de técnicas de alisado en dos ejemplares y pulido con cepillado en el tercero; mientras que para la superficie interna se empleó el pulido en una olla, cepillado en otra y en la tercera la superficie interna solo se alisó. Dentro de la muestra, no se observaron prácticas de decoración ni de tratamiento de superficie. Dos de las 


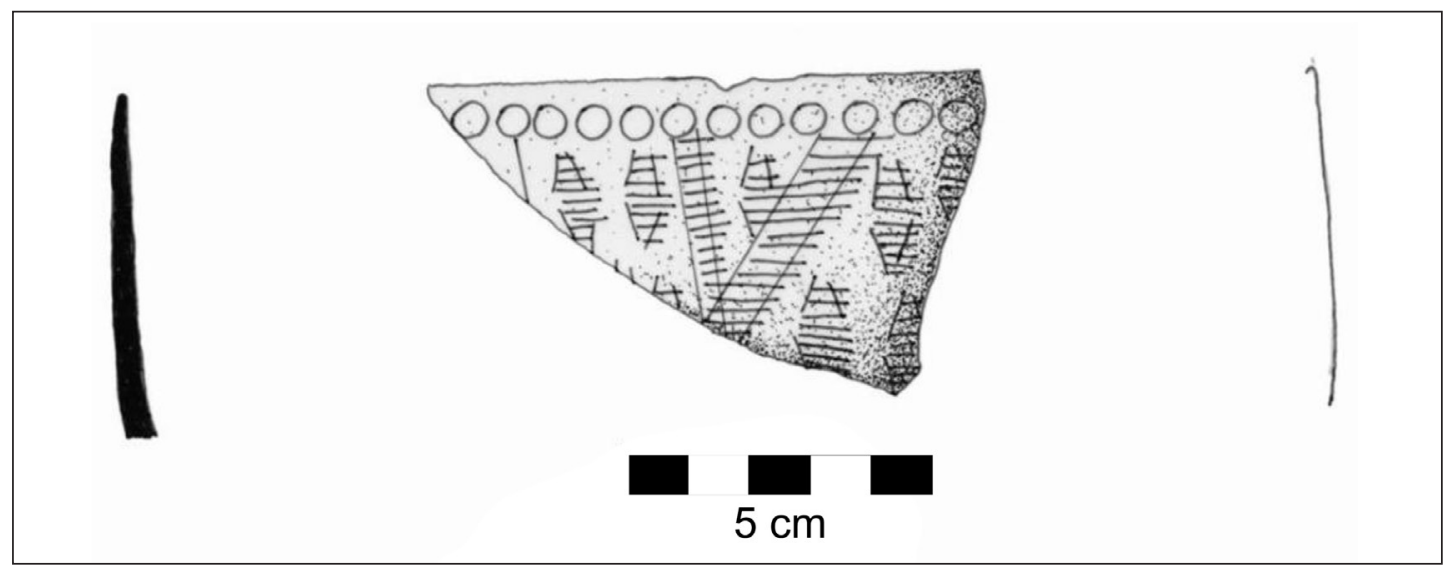

Figura 13. Fragmentos de un puco cerámico confeccionado con el tipo de Pasta B2 decorado mediante técnicas de incisión de motivos geométricos.

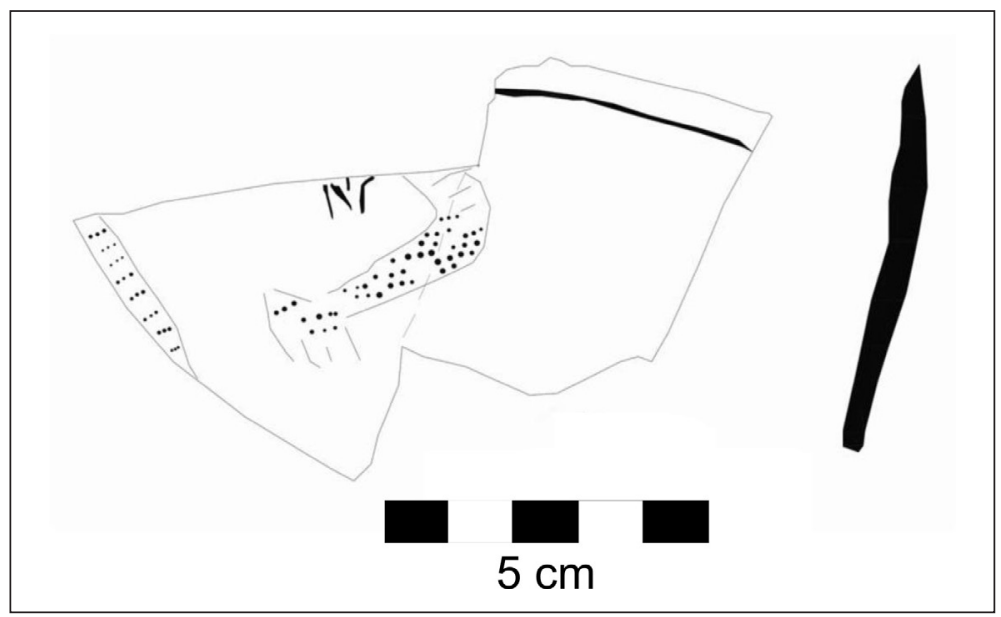

Figura 14. Fragmentos de un puco cerámico confeccionado con el tipo de Pasta B2 decorado mediante técnicas de incisión y escisión de motivos zoomorfos.

ollas fueron cocidas en atmósferas mixtas reductoras y la tercera en una atmósfera mixta oxidantes (Figura 12).

\section{Pasta B2}

Pucos o escudillas. Se pudo reconstruir un mínimo de cinco pucos dentro del grupo perteneciente a la pasta B2. Para su confección, se procedió al levantado de la pieza empleando las técnicas de modelado y rodete. Se observaron las técnicas de pulido en cuatro pucos y pulido con instrumento en el quinto, para el acabado de la superficie externa. Mientras que para la superficie interna se emplearon las técnicas de pulido con instrumento en tres de los pucos y en los dos restantes, alisado con instrumento y pulido. Solo en la superficie externa se observó técnicas decorativas por corte y/o extracción en cuatro pucos de la muestra de la Pasta B2 (Figuras 13 y 14). Las técnicas registradas fueron: incisión en dos ejemplares; incisión, escisión y estampamiento en los otros dos restantes. Los motivos de la decoración practicada en los pucos confeccionados con la Pasta B2 son: líneas y figuras zoomorfas; líneas y círculos; líneas, figuras geométricas y zoomorfas; y líneas y figuras geométricas. 
No se observó la presencia de técnicas de tratamiento de superficies. En lo que respecta al ambiente y tipo de cocción, se pudo determinar en todos los casos una cocción regular del material cerámico; así como dos tipos de cocciones: oxidantes en un puco y reductoras en los cuatro restantes.

\section{Pasta C}

Puco o escudilla. Dentro de la muestra analizada correspondiente a la Pasta $\mathrm{C}$, se pudo reconstruir la cantidad mínima de cinco pucos. Para levantarlo se emplearon técnicas de modelado y rodete. En cuanto al acabado de la superficie, se empleó la técnica de pulido de la superficie externa en todos los casos, y para la superficie interna se aplicó pulido en dos pucos y en los restantes; cepillado, pulido con instrumento y bruñido. Se observó decoración solo en la superficie externa y la misma se realizó por medio de la aplicación de las técnicas de corte; de incisión con estampamiento en dos pucos; y peinado con escisión en uno; y los otros dos por pintura monocromática roja. Los motivos observados corresponden a líneas cruzadas; líneas con surco rítmico (Figura 15); líneas y figuras geométricas; líneas y figuras zoomorfas; y círculos y figuras zoomorfas todas en iguales proporciones.

En un solo caso se observó una práctica de tratamiento de superficie mediante la aplicación de un baño en ambas superficies del puco. En cuanto al tipo y ambiente de cocción, se determinó la presencia de tres pucos con cocciones oxidantes, uno con cocción reductora, y otro con cocción reductora incompleta.

Ollas. Dentro de la muestra analizada del tipo de pasta C, solo se logró identificar la presencia de una olla, levantada mediante las técnicas de modelado y rodete. El acabado de la superficie externa se logró por pulido con instrumento; mientras que para la superficie interna se empleó el pulido. No se observó la aplicación de decoración, mientras que se realizó engobe en ambas superficies y su cocción se realizó en una atmósfera reductora y con una distribución regular.

\section{* Discusión}

En instancias previas, hemos podido detectar evidencias que refuerzan la idea de una manufactura local para los materiales cerámicos provenientes de Piedra Negra 02. A través de la comparación de los resultados de los análisis petrográficos de láminas delgadas de materiales cerámicos, con la litología de la zona, se pudo inferir el empleo de materias primas locales para la manufactura del conjunto cerámico estudiado (Espiro 2007, 2008). A su vez, la presencia de instrumentos asociados a la manufactura cerámica, como los alisadores cerámicos, puede considerarse como evidencias de prácticas manufactureras (Pozzi-Escot et al. 1993; Kojo 1996; Sapiencia de Zapata et al.1997). También se pudieron aislar técnicas e identificar aspectos del proceso de producción del conjunto de PIN O2, mientras se determinaron atributos referidos al uso brindado por los mismos dentro de la unidad doméstica. Las relaciones halladas entre las características de la pasta, el proceso de cocción, el tipo de decoración y el uso, nos llevaron a plantear una primera hipótesis sobre la existencia de una manufactura local y planificada de una gran parte de los materiales cerámicos empleados por los habitantes de la base residencial PIN O2 (Espiro 2006).

Luego de nuestro análisis, se hizo evidente que la Pasta A posee la mayor diversidad de formas (jarras o vasos, pucos y ollas, pipas e instrumentos) mientras que la Pasta B2 presenta la menor diversidad de formas (pucos e instrumentos). En lo que respecta a la forma de puco hemos observado muy pocas variaciones en torno a la sucesión de técnicas empleadas para su confección dentro de los cuatro tipos de pastas analizadas.

Existe una correlación entre los pucos de cualquier tipo de pasta y la presencia de cocciones regulares, lo cual puede ser interpretado como una evidencia de un ambiente de cocción controlado. Ahora bien, si tenemos en cuenta el concepto de elecciones tecnológicas brindado por Lemonnier (1992), hemos detectado en la muestra analizada las siguientes correlaciones: para la aplicación de la decoración en los pucos de los tipos de Pastas A, B2 y C, se identificó como ineludible el empleo de las técnicas de pulido y bruñido para el acabado de la superficie que iba a ser decorada, ya que bien la decoración se podría haber aplicado sobre la superficie alisada. También notamos que la aplicación de decoración por medio de técnicas de corte se corresponde con un tipo de cocción reductora, y que la decoración por agregado de pintura con el tipo de cocción oxidante. A su vez, los 


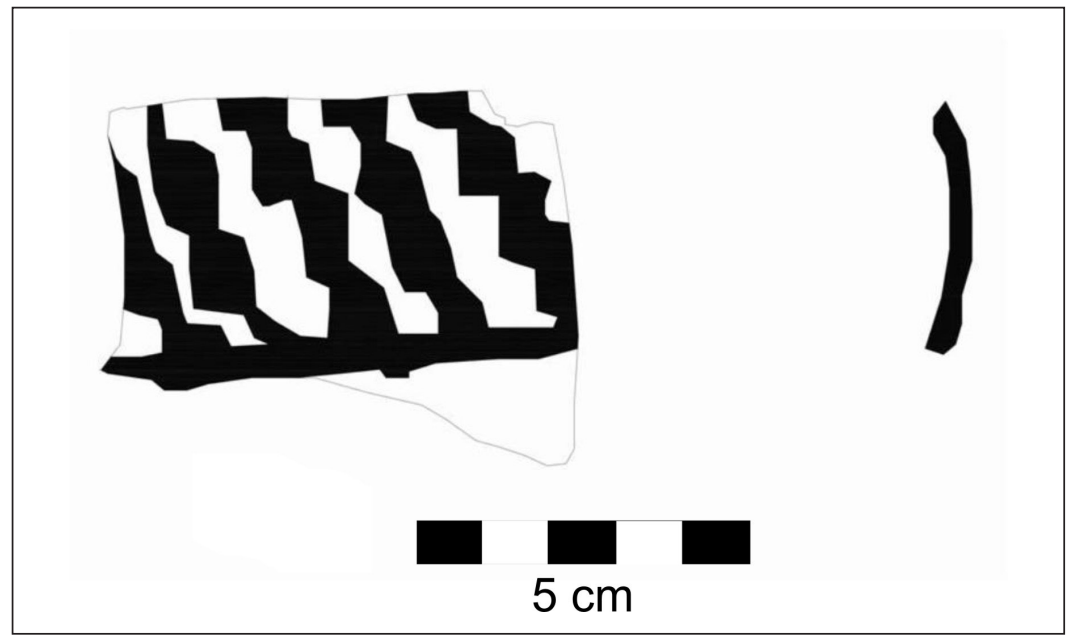

Figura 15. Fragmentos de un puco cerámico confeccionado con el tipo de Pasta $C$ decorado mediante el aplique de pintura negra formando líneas con surcos rítmicos.

pucos confeccionados con las Pastas A, B2 y C fueron sometidos a tratamientos de superficies por medio de engobes y baños.

En cuanto a las secuencias de procesos empleadas en la confección de ollas, hemos notado una mayor variabilidad de técnicas empleadas tanto hacia dentro de un mismo tipo de pasta como entre pastas distintas. Las ollas confeccionadas con las Pastas A y Br revelan cocciones irregulares, lo que puede estar indicando ambientes de cocción de las cerámicas no muy bien controlados. Únicamente algunos ejemplares confeccionados con el tipo de Pasta A poseen decoración en superficie por medio de técnicas de corte y notamos nuevamente la aplicación de pulido previo al decorado. Solo las ollas confeccionadas con los tipos de Pastas A y C poseen tratamiento de superficie de baños y engobes.

Podríamos encontrar una respuesta a estas diferencias si consideramos las asociaciones entre formas y posibles usos mencionados en la bibliografía clásica (Rice 1987; Skibo 1992). En este sentido suele ligarse a las formas de vasos o jarras y de pucos como las empleadas en el procesado y transferencia de alimentos y bebidas, mientras que las formas de ollas se vinculan a usos más versátiles, desde la preparación y cocción de alimentos, hasta el almacenamiento y transporte de comestibles y líquidos, y otras sustancias - secas o fluidas- no comestibles.
Siguiendo esta línea, en la Pasta C observamos características buenas para la exposición al fuego y cocción de alimentos. En este sentido, el único ejemplar de olla identificado para la Pasta $C$ presenta hollín en su superficie externa reforzando esta idea. ${ }^{6}$ La recurrencia del cepillado como técnica de acabado de superficie en las tres ollas confeccionadas con la Pasta Bı nos puede sugerir que las mismas fueron empleadas para el transporte o traslado de sustancias, ya que las superficies ásperas facilitan el levantado y traslado de las vasijas (Rice 1987). Un dato muy interesante asociado a las superficies disparejas es mencionado por Rice (1987), ya que aumenta la superficie para mantener propiedades térmicas o para evaporar líquidos.

Las ollas confeccionadas con la Pasta A presentan la mayor variabilidad, ya que algunos ejemplares presentan decoración y engobes o baños en sus superficies. En este sentido Rice (1987) y Skibo (1992) coinciden en que los baños y engobes ayudan a reducir la permeabilidad de las vasijas empleadas en el almacenamiento y procesamiento, si estos se presentan en su superficie externa producen un retardo en la penetración de líquidos; en cambio si ocurren en los interiores, disminuyen la penetración de escurrimiento y salpicado facilitando la limpieza de los recipientes.

${ }^{6}$ Muchos son los autores que coinciden en que la presencia de depósitos de hollín en una vasija es un claro indicador de su uso en la cocina y otras actividades que involucran el uso de fuego (Rice 1987; Rye 1988; Sinopoli 1991; Skibo 1992; Orton et al. 1993). 
Ahora bien, aunque nos parecen atractivas estas vías de explicación a la variabilidad observada, ellas no contemplan todos aquellos aspectos sociales, simbólicos que intervienen en la producción alfarera. Muchas veces las decisiones aparentemente arbitrarias en relación al desarrollo de una tecnología resultan lógicas dentro de la estructura social de una sociedad en particular (Loney 2000). Aquí podríamos mencionar las similitudes de forma y decoración entre todos los pucos de cocción reductora, independientemente del tipo de pasta con el que fueron confeccionados. O elección de un tipo de pasta con aptitudes para la exposición al fuego (Pasta C) en la manufactura de pucos que no poseen evidencias de tales alteraciones térmicas.

A esto último podemos agregar que dentro del conjunto se observaron ciertas acciones que no se relacionan con decisiones técnicas al momento de la manufactura de una vasija. En este sentido, la acción de reciclar una vasija fracturada (en un tortero, en un alisador, o en otra clase de instrumento) evidencia acciones - deliberadas o arbitrarias- de los habitantes de PIN O2 tendientes a mantener o conservar dentro de la esfera doméstica a ésta o parte de la misma. Tanto el reciclado como el mantenimiento de una alfarería retrasan el abandono o descarte de la misma, resultando en una conservación dentro de la esfera doméstica cotidiana, re-significándola y haciéndola partícipe en prácticas y relaciones, distintas para la que pudo haber sido confeccionada.

\section{$*$ Conclusiones}

Recordemos que el objetivo de identificar y describir las secuencias de procesos involucrados en la producción y manufactura de los materiales cerámicos de PIN oz es el de constituir un acercamiento preliminar para determinar la existencia de una tradición alfarera local en Laguna Blanca. En este sentido, podemos sugerir algunas de las diferencias que hemos observado en las secuencias de producción se sustentan en las decisiones que las/los ceramistas realizaron en función de brindarles a los materiales cerámicos propiedades o aptitudes para sus posibles usos. Otros aspectos se escapan a explicaciones tecno-prácticas. Quizá, parte de la variabilidad observada dentro de mismas formas se deba a la clasificación empleada en este trabajo, la cual responde a una racionalidad analítica y no a la racionalidad propia de quienes hicieron, contemplaron, usaron y le dieron significados a estos materiales cerámicos. De modo semejante, los objetos que nosotros clasificamos y agrupamos como cerámicos, debieron de haber respondido a otras clasificaciones y agrupamientos por parte de las personas que convivían con ellos.

Asimismo, los materiales cerámicos analizados del recinto A de PIN O2, no solo evidencian una participación en prácticas de reproducción del grupo (preparación y consumo de alimentos), sino también la participación en otras actividades (almacenaje, trasporte, actividades productivas, etc). La presencia de torteros o "muyunas" de cerámica, nos llevó a pensar en la realización de actividades relacionadas con la producción textil. La presencia de pipas cerámicas sugiere el consumo de psicotrópicos relacionados con vegetales que no son propios de la zona, como pueden haber sido el cebil (Anandenanthera columbrina) y/o tabaco (Nicotiana sp.). Los materiales cerámicos que oficiaron de ornamentos faciales (tembetaes y/o orejeras), junto con las cuentas de collar confeccionadas en piedra (algunas en malaquita), recuperados en la excavación, nos hablan de la participación de los materiales cerámicos en otra esfera de actividades dentro de la producción y reproducción del grupo doméstico, que no implica directamente su uso como recipiente de cocción o consumo.

En este trabajo hemos realizado una primera aproximación a la identificación de una tradición alfarera para tiempos del primer milenio DC en la región de Laguna Blanca y, no obstante haber identificado que parte de las variaciones en las secuencias de procesos se pueden entender en torno a una planificación de la manufactura, somos conscientes que debemos continuar trabajando en aquellas decisiones que nos pueden resultar arbitrarias o inconsistentes desde el punto de vista exclusivamente técnico. Creemos que, en estas últimas, podemos observar las relaciones a través de las cuales los materiales cerámicos fueron construidos socialmente por parte de los pobladores de la Aldea Piedra Negra y así poder determinar la existencia o no de una tradición alfarera local en Laguna Blanca.

Agradecimientos A los habitantes de Laguna Blanca y a los integrantes del Instituto Interdisciplinario Puneño (UNCa), por su colaboración y apoyo. También debo agradecer a los dos evaluadores de este trabajo, quienes lo enriquecieron con sus acertadas sugerencias y correcciones. 


\section{* Referencias citadas}

ALBECK, M. E., y M. C. SCATTOLÍN, 1984. Análisis preliminar de los asentamientos de Laguna Blanca (Catamarca) mediante el uso de la fotografía aérea. Revista del Museo de la Plata VIII: 279-302.

ARNOLD, D., 2003. Ecology and ceramic production in an Andean Community. New Studies in Archaeology. Cambridge University Press, Cambridge.

BATE, L. F., 1998. El Proceso de Investigación en Arqueología. Crítica Grijalbo, Barcelona.

CREMONTE, B., 1983-1985. Alcances y objetivos de los estudios tecnológicos en la cerámica arqueológica. Anales de Arqueología y Etnología 38-40: 179-216.

CONTRERAS CÓRTES, F., 1994. Una Aproximación a los estudios tipológicos. La Cerámica. Actas del II Congreso de Historia de Andalucía, 37-46, Córdoba, España.

DELFINO, D. D., 1997. Prospecciones en los '9o: Nuevas evidencias para repensar la arqueología de Laguna Blanca (Dpto. Belén. Catamarca). Revista de Ciencia y Técnica 7: 55-80.

2005. Entre la dispersión y la periferia. Sentido de presencias. Lagunización de La Aguada. En La cultura de La Aguada y sus expresiones regionales, pp. 263-291. EUDELAR. Museo de Ciencias Naturales, Universidad Nacional de la Rioja, La Rioja.

DELFINO, D. D., V. E. ESPIRO y R. A. DÍAZ, 2007. Excentricidad de las periferias: la región puneña de Laguna Blanca y las relaciones económicas con los Valles Mesotermales durante el primer milenio. En Procesos Sociales Prehispánicos en el Sur Andino. Producción y circulación de bienes, A. E. Nielsen, C. Rivolta, V. Seldes, M. Vázquez y P. Mercolli (Eds.), tomo II, pp. 167-190. Editorial Brujas, Córdoba.

DELFINO, D. D., V. E. ESPIRO y R. A. DÍAZ, 2012. Modo de vida, prácticas campesinas, comunidad y cosmovisión desde el primer milenio en Laguna Blanca (Dpto. Belén - Catamarca). En Precirculados del Encuentro de Arqueología del Período Formativo. Tafí del Valle, Tucumán.

ESPIRO, V. E., 2006. Aportes para una clasificación tecnológica de las cerámicas pertenecientes al Primer Milenio de nuestra era de la Aldea Piedra Negra, Laguna Blanca, Dpto. Belén, Provincia de Catamarca. Tesis de licenciatura. Escuela de Arqueología, Universidad Nacional de Catamarca, Catamarca.

2007. Materiales cerámicos y los pobladores de Laguna Blanca durante el primer milenio D.C. Aportes Científicos desde Humanidades 7: 14-21.
2008. Características del Proceso de Manufactura de las Alfarerías de La Aldea Piedra Negra, correspondientes al primer milenio de nuestra era, Distrito Laguna Blanca, Departamento Belén, Provincia de Catamarca. La Zaranda de Ideas, Revista de Jóvenes Investigadores en Arqueología 4: 9-25.

GONZÁLEZ, A. R., 1955. Investigaciones arqueológicas en el N.O. argentino. Ciencia e Investigación 10 (7):322-325.

1961-1964. La Cultura de La Aguada del N.O. Argentino. Revista del Instituto de Antropología de Córdoba 2-3: 205-252.

1963. Cultural development in northwestern Argentina. En Aboriginal Cultural Development in Latin America: an Interpretative Review, Smithsonian Miscellaneous Collections 146 (1): 103-117.

1979. La dinámica cultural del N.O. argentino. Evolución e historia en las culturas del N.O. argentino. Antiquitas 28-29: 1-15.

KOJO, Y., 1996. Production of Prehistoric Southwestern ceramics: a low-technology approach. American Antiquity 61 (2):325-339.

LEMONNIER, P., 1992. Elements for an anthropology of technology. Anthropological Papers 88: 1-24.

LONEY, H., 2000. Society and technological control: A critical review of models of technological change in ceramic studies. AmericanAntiquity 65 (4): 646-668.

MANZANILLA, L., 1986. Unidades habitacionales mesoamericanas y sus áreas de actividad. IIA/UNAM, México D.F.

NÚÑEZ, L., y T. DILLEHAY, 1995. movilidad giratoria, armonía social y desarrollo en los Andes Meridionales: Patrones de tráfico e interacción económica. Universidad Católica del Norte, Antofagasta.

POZZI-ESCOT, D., M. ALARCÓN y C. VIVANCO, 1993. Instrumentos de alfareros de la época Wari. Bulletin Institute Frances de Études Andines 22 (2): 467-496.

ORTON, C., P. TYERS y A. VINCE, 1993. Pottery in Archaeology. Cambridge Manuals in Archaeology, Cambridge University Press, Cambridge.

RICE, P., 1987. Pottery Analysis. University of Chicago Press, Chicago.

RYE, O., 1988 [1981]. Pottery Technology. Principles and Reconstruction. Manuals on Archaeology 4. Taraxacum, Washington.

SAPIENCIA DE ZAPATA, S., V. MACEDA RASSIT y J. VIAÑA UZIEDA, 1997. Inventario de la cerámica aymara y quechua. Unión de Ceramistas Aymaras y Quechuas de Bolivia. Centro de Investigación de Energía y Población, La Paz. 
SCATTOLÍN, M. C., y M. F. BUGLIANI, 2005. Un repertorio surtido: las vasijas del oasis de Laguna Blanca, Puna argentina. Revista Española de Antropología Americana 35: 51-74.

SHENNAN, S., 1992. Arqueología Cuantitativa. Editorial Crítica, Barcelona.

SHEPARD, A., 1968. Ceramics for the Archaeologist. Sith Printing Publication 609. Carnegie Institution of Washington, Washington.

SINOPOLI, C., 1991. Approaches to Archaeological Ceramics. Plenum Press, New York.

SKYBO, J. M., 1992. Pottery Function. A Use-Alteration Perspecive. Plenum Press, New York.
TARRAGÓ, M. N., 1984. La historia de los pueblos circumpuneños en relación con el Altiplano y los Andes Meridionales. Estudios Atacameños 7: 116-132.

TURNER, J. C., 1973. Descripción de la Hoja 11d, Laguna Blanca. Provincia de Catamarca. Carta Económico-Geológica de la República Argentina Escala 1: 200.000. Ministerio de Industria y Minería, Subsecretaría de Minería. Servicio Nacional Minero Geológico, Buenos Aires.

ZAGORODNY, N., 2000. Descripción de una técnica expeditiva de análisis cerámico. Contribución Arqueológica 5: 259-266.

ZAGORODNY, N., y B. BALESTA, 1999. La construcción de grupos de referencia como herramienta en la investigación ceramológica. Actas del XII Congreso Nacional de Arqueología Argentina, tomo II: 55-62. 